\title{
Geometric Structures in Hadronic Cores of Extensive Air Showers Observed by KASCADE
}

\author{
T. Antoni ${ }^{1}$, W.D. Apel ${ }^{2}$, F. Badea ${ }^{2}$ K. Bekk ${ }^{2}$, A. Bercuci ${ }^{3}$, J. Blümer ${ }^{2,1}$, H. Bozdog ${ }^{2}$, I.M. Brancus ${ }^{3}$, \\ A. Chilingarian ${ }^{4}$, K. Daumiller ${ }^{2}$, P. Doll ${ }^{2}$, R. Engel $^{2}$, J. Engler ${ }^{2}$, F. Feßler ${ }^{2}$, H.J. Gils ${ }^{2}$, R. Glasstetter ${ }^{1} \dagger$ \\ A. Haungs ${ }^{2}$, D. Heck ${ }^{2}$, J.R. Hörandel ${ }^{1}$, K-H. Kampert ${ }^{1,2}$ 亓 H.O. Klages ${ }^{2}$, G. Maier ${ }^{2}$ 团 H.J. Mathes ${ }^{2}$, \\ H.J. Mayer ${ }^{2}$, J. Milke ${ }^{2}$, M. Müller ${ }^{2}$, R. Obenland ${ }^{2}$, J. Oehlschläger ${ }^{2}$, S. Ostapchenko ${ }^{2}$ M. Petcu ${ }^{3}$, \\ H. Rebel ${ }^{2}$, A. Risse ${ }^{5}$ M. Risse ${ }^{2}$, M. Roth ${ }^{1}$, G. Schatz ${ }^{2}$, H. Schieler ${ }^{2}$, J. Scholz ${ }^{2}$, T. Thouw ${ }^{2}$, \\ H. Ulrich ${ }^{2}$, J. van Buren ${ }^{2}$, A. Vardanyan ${ }^{4}$, A. Weindl ${ }^{2}$, J. Wochele ${ }^{2}$, and J. Zabierowski ${ }^{5}$
}

(1) Institut für Experimentelle Kernphysik, Universität Karlsruhe, 76021 Karlsruhe, Germany

(2) Forschungszentrum Karlsruhe, Institut für Kernphysik, 76021 Karlsruhe, Germany

(3) National Institute of Physics and Nuclear Engineering, 7690 Bucharest, Romania

(4) Cosmic Ray Division, Yerevan Physics Institute, Yerevan 36, Armenia

(5) Soltan Institute for Nuclear Studies, 90950 Lodz, Poland

\begin{abstract}
The geometric distribution of high-energy hadrons $\geq 100 \mathrm{GeV}$ in shower cores measured with the KASCADE calorimeter is analyzed. The data are checked for sensitivity to hadronic interaction features and indications of new physics as discussed in the literature. The angular correlation of the most energetic hadrons and in particular the fraction of events with hadrons being aligned are quantified by means of the commonly used parameter $\lambda_{4}$. The analysis shows that the observed $\lambda_{4}$ distribution is compatible with that predicted by simulations and is not linked to an angular correlation from hadronic jet production at high energy. Another parameter, $d_{4}^{\max }$, describing distances between hadrons measured in the detector, is found to be sensitive both to the transverse momenta in secondary hadron production and the primary particle type. Transverse momenta in high-energy hadron interactions differing by a factor two or more from what is assumed in the standard simulations are disfavoured by the measured $d_{4}^{\max }$ distribution.
\end{abstract}

PACS numbers: 96.40.Pq,96.40.-z,13.85.-t,13.85.Tp

\section{INTRODUCTION}

High-energy hadrons in extensive air showers offer a unique possibility to study interaction features well beyond the kinematic and energy region of earthbound accelerators. In particular, structures in hadronic shower cores might reflect properties of the particle production at an initial stage of the shower development. For example, the EAS-TOP Collaboration investigated multicore events recorded in the calorimeter and studied the cross-section of large $p_{t}$ jet production in proton-air collisions [1]. In other studies, data taken by the KASCADE scintillator array and hadron calorimeter [2, 3] were used to investigate different aspects of hadronic interaction models. The correlation of the hadronic to the muonic and electromagnetic shower components [4, 5], as well as features of the particle production in the very forward region of nucleon-air collisions $[\underline{6}]$ were measured and compared to model predictions.

Geometric structures in hadronic shower cores at ob-

\footnotetext{
${ }^{*}$ On leave of absence from (3)

${ }^{\dagger}$ Now at University of Wuppertal, 42117 Wuppertal, Germany

¥Now at University of Leeds, LS2 9JT Leeds, UK

$\S$ On leave of absence from Moscow State University, 119899 Moscow, Russia

๑Corresponding author. Electronic address: iwan@zpk.u.lodz.pl
}

servation level are particularly interesting, as one expects QCD jet production to lead to secondary hadrons being naturally aligned to form line shape patterns [7]. Similar alignment structures might result from exotic hadron production processes [8]. Therefore, it is not surprising that the observation of aligned structures in a number of emulsion chamber experiments has initiated considerable experimental and theoretical efforts over the last two decades 7, 8, 9, 10, 11, 12, 13, 14, 15, 16, 17, 18, 19, 20, 21, 22, 23, 24, 25, 26].

Aligned event structures were, for instance, reported by the PAMIR experiment [9, 10. An excess of events with substructure alignment above background fluctuations was found in the data for primary energies above an energy threshold of $8-10 \mathrm{PeV}$ (see e.g. 10, 11, 12, 13, 14, 15] and references therein). The most energetic event of $\simeq 10 \mathrm{PeV}$ that was measured in an emulsion experiment during flights with the Concorde airplane also shows alignment [16]. Furthermore, alignment was found in another individual high-energy event, estimated to have an energy $>10 \mathrm{PeV}$, that was detected by a balloon borne emulsion chamber [17].

The experimental evidence of the existence of an alignment phenomenon, i.e. events with substructures being located in a line occurring more frequently than expected, is debated controversially, including the possibility of the excess being due to statistical fluctuations (see e.g. [16]).

At lower energy, no excess of aligned structures in air 
showers or direct experiments was observed. At energies of a few $\mathrm{PeV}$ the fraction of elongated events measured by PAMIR was found to agree with the expectations from background fluctuations. The fraction of elongated events of similar energy recorded by the Kanbala air shower experiment [18] gave also no indication of a significant excess over the simulated background. Measurements of the balloon experiment RUNJOB in a primary energy range of $0.01-0.1 \mathrm{PeV}$ have shown that the fraction of aligned events agreed with the background expectation [19]. Also the less energetic events recorded during the Concorde flights showed no excess alignment.

A search for such alignment phenomena at accelerators was performed at comparatively low collision energy of $250 \mathrm{GeV}$ in $\pi-\mathrm{Au}$ interactions. No significant excess of elongated events was found in data of the CERN experiment NA22 [10, 20].

Various theoretical efforts, partly hampered by the limited experimental guidance, were performed to explain alignment by physics mechanisms. It was pointed out in [7] that dynamical features of standard QCD jet production might give rise to observable alignment. In 10, 15 it was emphasized that the observations would indicate aligned particle production in the fragmentation region of the collision rather than in the central region. A connection of alignment to the production of secondaries with high transverse momentum was suggested e.g. in 13, 14. The appearance of an anti-shadow scattering mode at high collision energies as source of alignment was discussed in [21]. An onset of semi-hard double inelastic diffraction was proposed in [8], where alignment results as projection from the rupture of a quark-gluon string. The existence of a new particle with a long mean free path, produced in a new type of interaction, was also proposed to explain the PAMIR observation 15, 22].

Simulations based on phenomenological models were compared to the PAMIR data in [14, 23]. It was pointed out that alignment features produced, e.g., in the first interaction of the primary cosmic ray in air can be washed out by subsequent interactions during the shower evolution.

While the existence of alignment features is out of doubt, the main questions concerning their nature are: To what extent can such structures observed in the data be related to specific particle production and interaction mechanisms? Are new particles or interaction channels, maybe above a certain collision energy threshold, required to explain the data?

In this work, geometric structures in hadronic shower cores recorded by the KASCADE experiment are investigated. Both, the alignment of hadrons in shower cores and the distance between hadrons are analyzed and compared to detailed Monte Carlo simulations.

The article is organized as follows. In Section III relevant features of the KASCADE detector and the applied data selection are described. Results on aligned geometric structures are presented in Sec. IIII including a detailed study of the background expectation due to random fluctuations. Another observable, characterizing the distance between hadrons, is introduced in Sec. IV and used to analyze KASCADE data. Implications and comparisons to results from other experiments are discussed in Sec. D

\section{MEASUREMENTS}

The KASCADE air shower experiment [2] is located at an altitude of $110 \mathrm{~m}$ a.s.l. (corresponding to $1020 \mathrm{~g} \mathrm{~cm}^{-2}$ atmospheric depth) at Karlsruhe, Germany. Its $16 \times 20 \mathrm{~m}^{2}$ hadron calorimeter [3] is well suited to investigate geometric structures in hadronic shower cores. Individual hadrons with energies from $50 \mathrm{GeV}$ up to more than $20 \mathrm{TeV}$ are measured with a spatial resolution of $\simeq 15 \mathrm{~cm}$. The primary energy covered by KASCADE includes the range of 8-10 PeV claimed as threshold energy for alignment. A detailed description of the KASCADE detector performance and the standard reconstruction procedures for the array and calorimeter data is given in [2, 3].

For the analysis, data collected by the KASCADE experiment in the period from May 1998 to April 2001 have been used. Primary energy, direction, and core position of air showers with energies $>0.5 \mathrm{PeV}$ are determined with the scintillator array data. The energy, position and direction of individual hadrons with energies $\geq 50 \mathrm{GeV}$ are reconstructed from the measurements performed with the hadron calorimeter. The following selection criteria are applied to the data:

(i) The reconstructed primary energy of the air shower $\left(\Delta E_{0} / E_{0} \simeq 25 \%\right)$ is $\geq 1 \mathrm{PeV}$, and the primary zenith angle is $\leq 30^{\circ}\left(\Delta \theta \simeq 1^{\circ}\right)$.

(ii) The reconstructed shower core location $\left(\Delta x_{c} \simeq 1.5 \mathrm{~m}\right)$ is contained in the active calorimeter area with a minimum distance to the calorimeter boundaries of $3 \mathrm{~m}$.

(iii) At least four hadrons with energies $\geq 100 \mathrm{GeV}$ are reconstructed $\left(\Delta E_{h} / E_{h} \simeq 20 \%\right)$.

The effect of the latter condition is small even at lower primary energies, reducing the data set by $\simeq 13 \%$.

In total, 4489 events are selected this way. After transformation into the shower plane, the quantities $\lambda_{4}$ and $d_{4}^{\max }$ (see below) are evaluated for each event. An example of a measured elongated event is given in Fig. 1]

\section{ALIGNMENT OF HADRONS}

Commonly, the degree of alignment is described by a parameter $\lambda_{4}$ [24] quantifying the angular correlation between the four most-energetic particles (or particle clusters):

$$
\lambda_{4}=\frac{1}{24} \cdot \sum_{i \neq j \neq k}^{4} \cos 2 \varphi_{i j}^{k}
$$




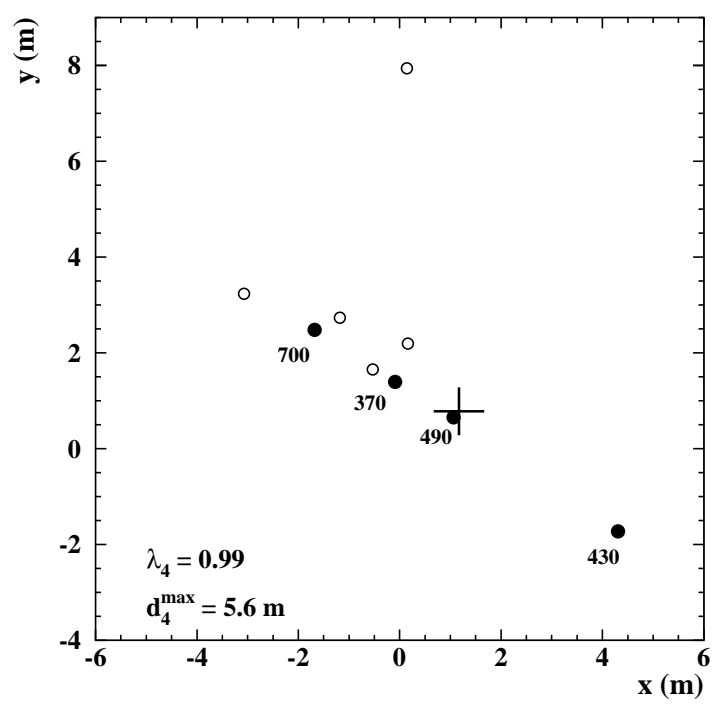

FIG. 1: Example of a measured hadronic shower core (hadron positions in the shower plane) with $\lambda_{4}=0.99$ and $d_{4}^{\max }=$ $5.6 \mathrm{~m}$. 9 hadrons with energies above $100 \mathrm{GeV}$ are reconstructed. For the four most energetic hadrons (full symbols), the energies in $\mathrm{GeV}$ are given. The shower core position as reconstructed by the scintillator array is marked by a cross. The active calorimeter area exceeds the area plotted.

where $\varphi_{i j}^{k}$ denotes the angle between the lines connecting particle $k$ to $i$ and $j$. Possible values range between $\lambda_{4}=-\frac{1}{3}$ (isotropic distribution) and $\lambda_{4}=1$ (perfect alignment). Events are usually termed "aligned" or "elongated" for $\lambda_{4} \geq 0.8$.

The measured $\lambda_{4}$ distribution is displayed in Fig. 2 Also shown are the results for primary proton and iron nuclei simulated with the CORSIKA (v6.0) code 27] employing the QGSJET 01 28] hadronic interaction model. The simulations are performed for a continuous primary energy spectrum with a spectral index -2.7 . It has been checked explicitely that adopting other values for the primary energy slope or, e.g., including a knee structure does not influence the calculated $\lambda_{4}$ distribution, as will be also clear from the following discussion. The shower simulation is followed by a detailed detector simulation based on GEANT [29]. The same reconstruction algorithms and selection criteria are applied to simulations and data. The simulation statistics is of comparable size to the data statistics for each primary.

Fig. 2 shows that the measured $\lambda_{4}$ distribution and the fraction of aligned events are well reproduced by the standard calculations, without the need of introducing new particles or non-standard interaction features. Moreover, despite the much smaller initial energy per nucleon in case of iron showers, no significant difference between proton and iron induced events is observed. This already indicates a minor sensitivity of the observed parameter $\lambda_{4}$ to hadronic particle production in the first interactions.

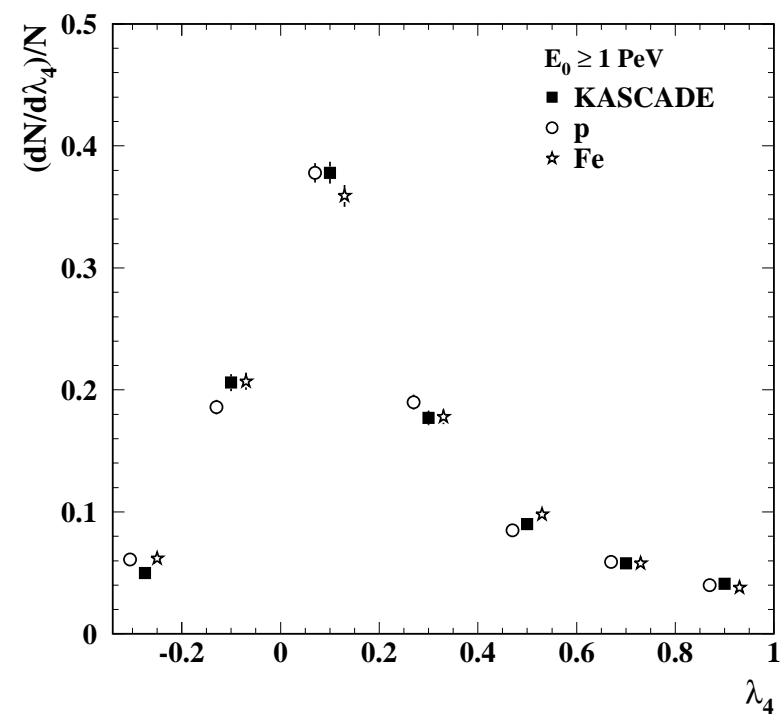

FIG. 2: $\lambda_{4}$ distribution measured by KASCADE compared to simulation results for primary proton and iron showers. For clarity, simulation points are slightly displaced horizontally.

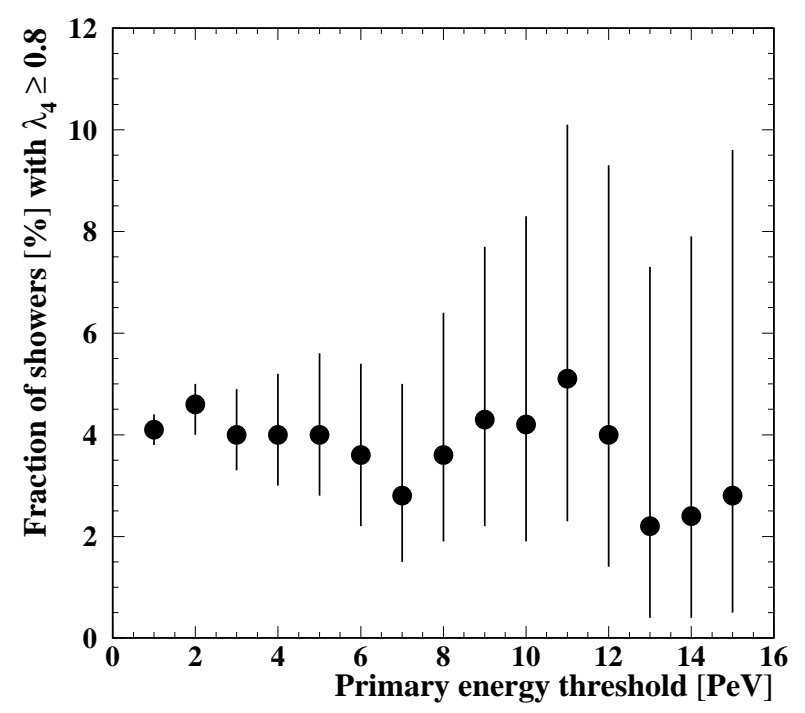

FIG. 3: Fraction of aligned events with $\lambda_{4} \geq 0.8$ as measured by KASCADE versus the primary energy threshold.

The measured fraction of aligned events $\left(\lambda_{4} \geq 0.8\right)$ is plotted in Fig. 3 as a function of the primary energy threshold for selecting showers. Within the statistical uncertainties, no energy dependence of the fraction of elongated events is observed. In particular, no significant increase or threshold behaviour for higher primary energies is found.

In the following, the sensitivity of the observed $\lambda_{4}$ distribution and the fraction of aligned event structures 
to specific particle production features is investigated. Events with aligned hadronic structures could be the result of jet production in hadronic interactions during the shower development [7]. The jet pair and the hadrons from projectile fragmentation are naturally aligned in one plane and the expected geometric separation falls in the range measurable with KASCADE. For example, the projected separation from the initial direction of a $10 \mathrm{TeV}$ hadron produced at an altitude of $15 \mathrm{~km}$ with a transverse momentum $p_{t}=5 \mathrm{GeV}$ is $7.5 \mathrm{~m}$ at KASCADE observation level.

To investigate the correlation between the angular distribution of particles in hadronic interactions and geometric structures, a simulation was carried out in which the azimuth angles of the produced secondary particles are picked at random in the centre-of-mass system of the collision, keeping the transverse and longitudinal momenta unchanged. In this way, any angular correlation of produced particles is eliminated. The results of calculations performed for $5 \mathrm{PeV}$ primary protons are shown in Fig. 4 together with the standard simulations. No significant change of the distribution as a whole or the fraction of aligned events is observed for the modified version (labeled " $\Phi_{\text {random }}$ ") with respect to the original one.

Next, the sensitivity of $\lambda_{4}$ to the transverse momentum $p_{t}$ of the secondaries is checked. A connection of high$p_{t}$ events and alignment was suggested, e.g., in [13, 14]. In the simulation, the $p_{t}$ of each secondary produced in a hadronic interaction is artificially increased by a factor two (labeled " $2 \cdot p_{t}$ " in Fig. 4), keeping the original angular correlations unchanged. Such an increase is not realistic, but used here to study the sensitivity. The overall shape of the $\lambda_{4}$ distribution is affected very little. No significant change of the fraction of aligned events can be noted.

This insensitivity to modifications of the hadronic particle production suggests that the measured $\lambda_{4}$ distribution is mostly due to a random distribution of the hadron azimuth angles. Thus, a simplified simulation is performed by artificially generating sets of four hadron positions in the shower plane. The azimuth angles of the hadrons are chosen randomly with respect to the shower core. The lateral distances of the hadrons are sampled according to the measured lateral distribution of the four most energetic hadrons. The KASCADE data are indeed reproduced, see Fig. 5

The sensitivity of the $\lambda_{4}$ distribution to the shape of the lateral distribution of the observed particles is checked with the simplified simulation by varying the assumed lateral shape, keeping the choice of hadron azimuth angles at random. It should be noted that $\lambda_{4}$ as an observable, being determined from the event topology, does not depend on the absolute length scale of the assumed lateral distribution, but only on its shape. As can be seen in Fig. [6] even choices of functional forms that are unrealistic for air shower lateral distribution such as adopting a particle density that is constant for any distance from the core, result only in modest changes of the

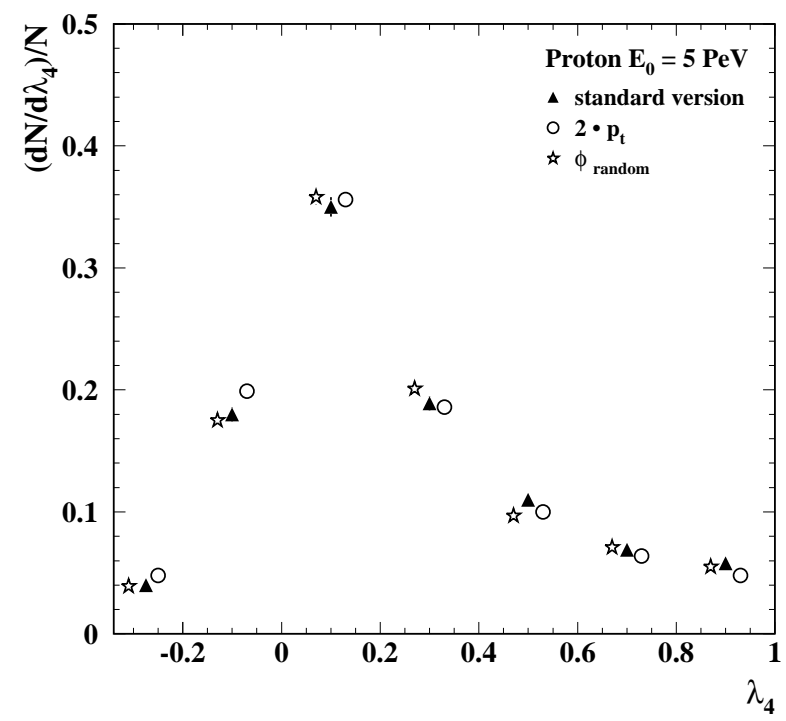

FIG. 4: $\lambda_{4}$ distributions for proton showers of $5 \mathrm{PeV}$ primary energy without modification of the hadronic particle production ("standard version"), with randomly chosen azimuth angles of secondary hadrons (" $\Phi$ random"), and with transverse momenta of secondary hadrons increased by a factor two (" $2 \cdot p_{t}$ "). Simulation points obtained after the modifications are slightly displaced horizontally.

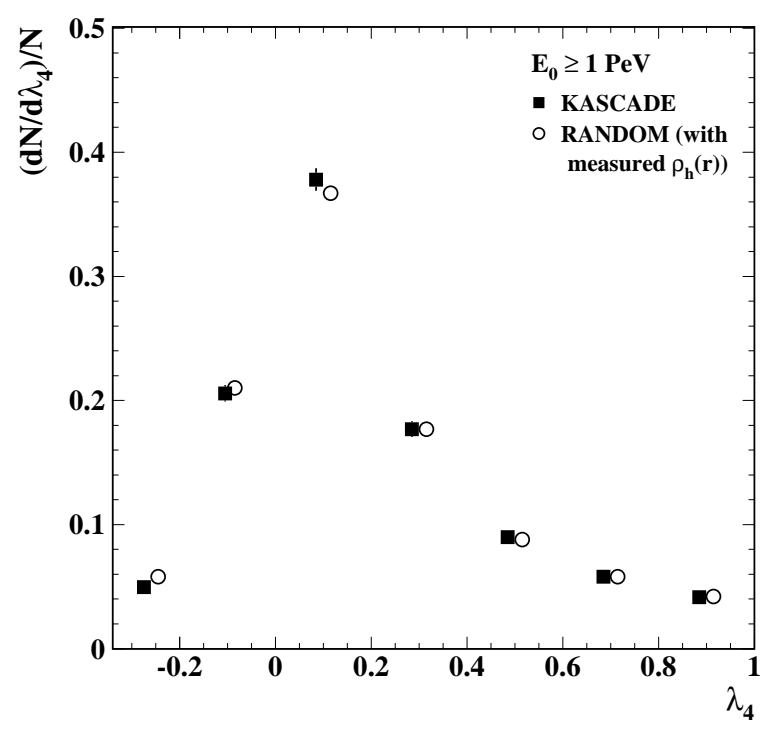

FIG. 5: $\lambda_{4}$ distributions: KASCADE data and results from a simplified simulation of event topologies, in which the azimuth angles were chosen randomly with respect to the shower core, and the distances according to the measured hadron lateral distribution (see text). Simulation points are slightly displaced horizontally. 


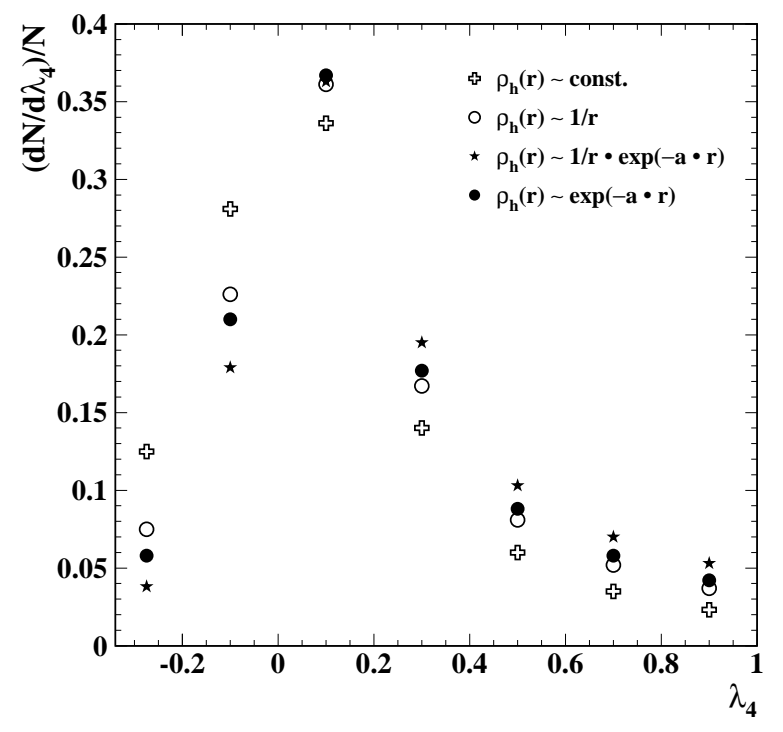

FIG. 6: $\lambda_{4}$ distributions obtained by a simplified simulation of event topologies (see text or caption of Fig. 5) for different hypothetical shapes of the lateral distribution as indicated.

$\lambda_{4}$ distribution. In general, a flatter lateral distribution tends to decrease the fraction of aligned events generated in the random sample. However, the sensitivity of $\lambda_{4}$ to different lateral shapes is minor. It seems hardly possible for an air shower experiment to observe differences between $\lambda_{4}$ distributions that are due to different lateral distributions of the measured particles only.

Other parameters describing geometric structures similar to $\lambda_{4}$ have been checked for sensitivity to primary mass or hadronic interaction characteristics [30]. For instance, all hadrons $\geq 100 \mathrm{GeV}$ instead of the four most energetic ones are considered to calculate a generalized quantity $\lambda_{n}$. Also, weight factors for the hadrons are introduced to additionally take their reconstructed energy into account. No significant sensitivity to hadronic particle production properties of these $\lambda$-related quantities is found, either.

\section{DISTANCE BETWEEN HADRONS}

Hadronic shower observables with sensitivity to interaction features have for instance been discussed in [4, $[\underline{6}]$. A specific, further observable that is based on the four most energetic hadrons in each event as is $\lambda_{4}$, is given by the maximum distance, $d_{4}^{\max }$, between one of the four considered hadrons to the geometric centre of the other three [26]. Since this quantity is intimately connected to the hadron lateral distribution, a sensitivity both to hadronic interaction features such as the transverse momentum of secondary hadrons and to the primary particle type can be expected.

The $d_{4}^{\max }$ distribution measured by KASCADE at en-

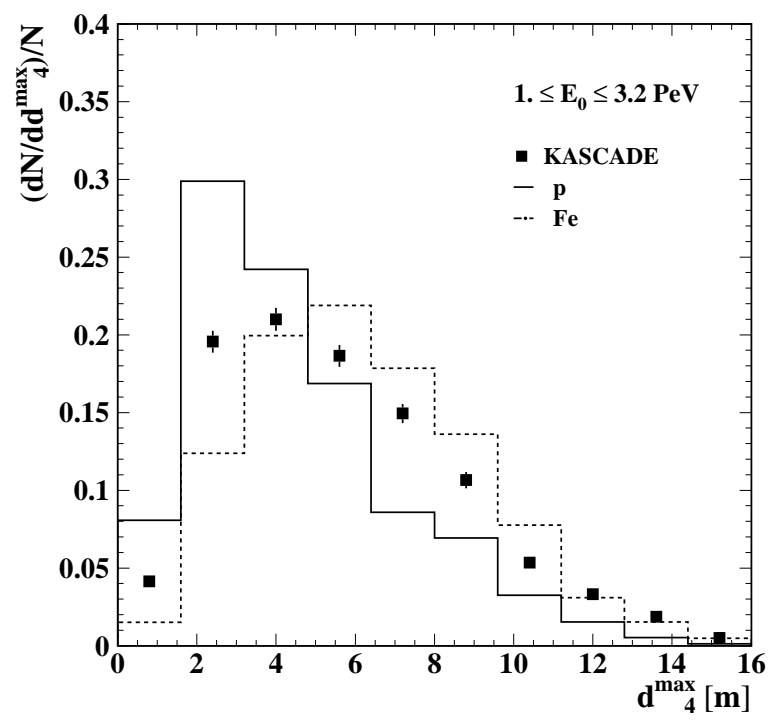

FIG. 7: $d_{4}^{\max }$ distribution measured by KASCADE compared to simulation results for primary proton and iron showers for primary energies of $1-3.2 \mathrm{PeV}$.

ergies 1-3.2 PeV is compared to simulation results for primary proton and iron showers in Fig. 7 The comparison is restricted to energies below the knee due to the increasing uncertainty of primary flux spectra and composition at higher energy. The $d_{4}^{\max }$ distribution for primary protons is peaked at smaller values compared to the predictions for iron-initiated events, reflecting a steeper hadron lateral distribution in case of air showers initiated by proton primaries.

The KASCADE data are mostly bracketed by the primary proton and iron expectations. This seems reasonable, as for these primary energies a mixed composition is favoured from extrapolations of direct cosmic-ray measurements, from other air shower experiments and in particular from the analysis of independent KASCADE observables such as the electron and muon shower sizes [31]. Only at largest $d_{4}^{\max }$ values, a slight underestimation shows up in the predictions that may indicate shortcomings in the modelling of hadron interactions.

In the next step, as before, the $p_{t}$ of secondary hadrons is artificially modified in the simulations. The results are compared to KASCADE data in Fig. 8 Assuming transverse momenta of secondary hadrons produced in highenergy interactions twice as large as in standard simulations (upper panel in Fig. 8), the distributions both for proton and iron primaries are significantly shifted to larger $d_{4}^{\max }$ values. In this scenario, the simulations are hardly able to provide a satisfactory description of the $d_{4}^{\max }$ data. For any assumption on the primary composition between proton and iron nuclei, and in particular for a mixed composition as favoured by other measurements, events with small values of $d_{4}^{\max }$ observed in hadronic shower cores are not adequately reproduced by simula- 

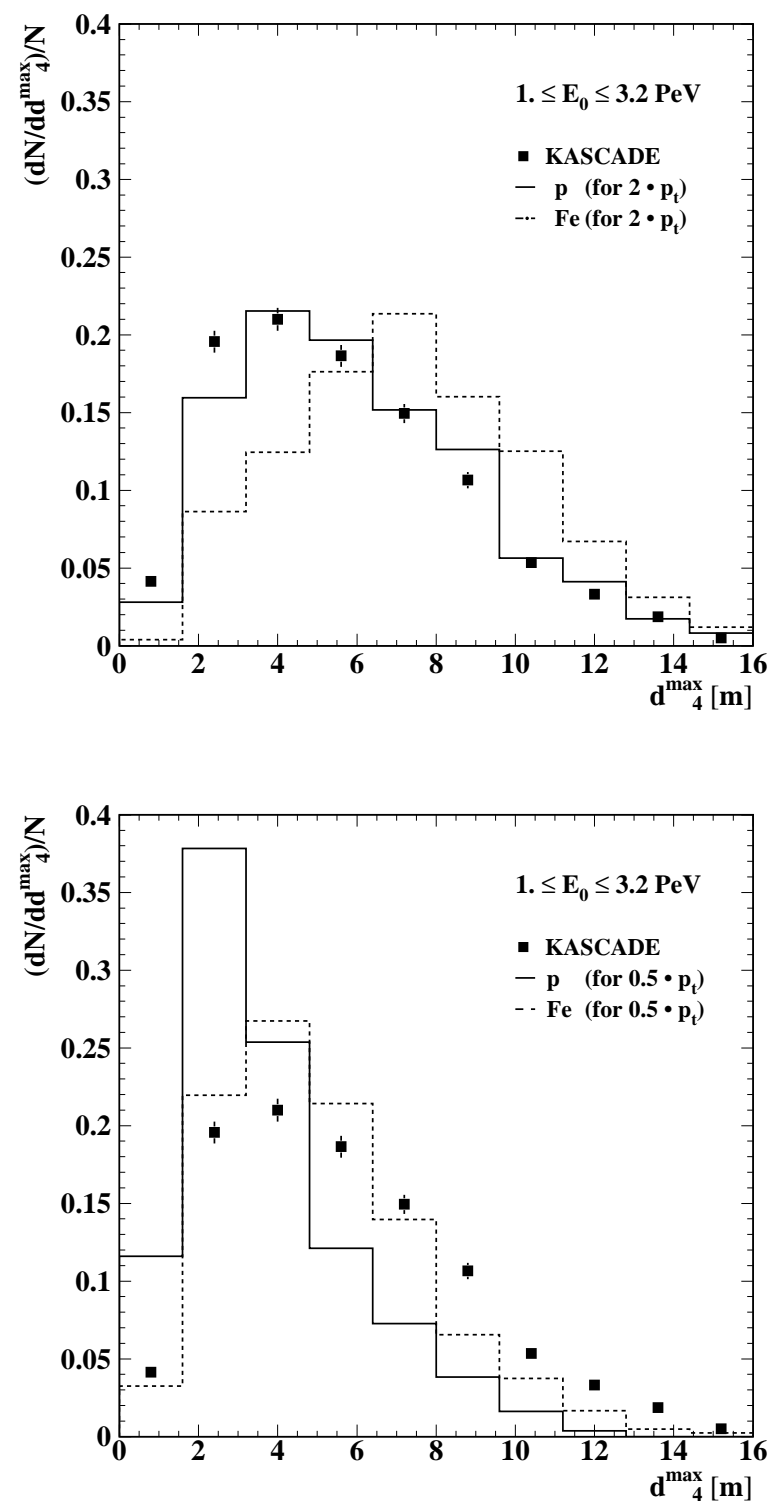

FIG. 8: $d_{4}^{\max }$ distribution measured by KASCADE (see Fig. (7) compared to simulation results for primary proton and iron showers with modified transverse momentum of secondary hadrons. The transverse momenta were artificially increased (upper panel, " $2 \cdot p_{t}$ ") and reduced (lower panel, " $0.5 \cdot p_{t}$ ") by a factor two.

tions with doubled $p_{t}$. The same conclusion holds when artificially reducing the $p_{t}$ of secondary hadrons by a factor two (lower panel in Fig. 8). In this case, the simulations fail to describe the events observed with large values of $d_{4}^{\max }$. Therefore, hypothetical transverse momenta in high-energy secondary hadron production that differ by a factor two or more from the standard assumptions are disfavoured by the KASCADE data.

Another application of the $d_{4}^{\max }$ parameter is related to investigating the primary cosmic-ray composition by means of air shower simulations. Due to the sensitivity to the primary particle type, quantities such as $d_{4}^{\max }$ can be used to check the internal consistency of hadronic interaction models.

\section{DISCUSSION}

The results of the KASCADE data analysis concerning the $\lambda_{4}$ parameter can be summarized as follows. The measured $\lambda_{4}$ distribution and the fraction of aligned events of high-energy hadrons are well reproduced by the simulations. Moreover, the data follow the expectations from randomly distributed hadron azimuth angles. No significant dependence on the primary energy was observed. Within the statistical uncertainty, no correlation between $\lambda_{4}$ and hadronic interaction features such as jet production was found.

These results are not necessarily in contradiction to possible alignment excesses in other shower observables, at different observation levels, or in a different primary energy range. However, the absence of an alignment excess in the present analysis might be used to constrain attempted alignment explanations.

The KASCADE data can be compared to the $\lambda_{4}$ distribution measured by the PAMIR detector. PAMIR [9, 10] is an emulsion chamber experiment located at an altitude of $4360 \mathrm{~m}$ a.s.l. (corresponding to $600 \mathrm{~g} \mathrm{~cm}^{-2}$ atmospheric depth), measuring air shower hadrons and photons with a particle energy threshold of a few TeV.

In Fig. 9] the $\lambda_{4}$ distributions obtained from KASCADE and PAMIR data 11, 15 are compared for two different primary energies. In the energy range of a few $\mathrm{PeV}$ (upper panel of the Figure), i.e. below the threshold energy of the alignment excess claimed from PAMIR data, both distributions and the fraction of aligned events agree well to each other. This is a quite significant result, since in the PAMIR experiment, compared to KASCADE, a completely different detection technique is applied, the mass overburden is considerably smaller $\left(\Delta X_{v} \simeq 420 \mathrm{~g} \mathrm{~cm}^{-2}\right)$, and the energy threshold of the registered shower particles is higher by more than an order of magnitude. A possible interpretation of this concordance is that both $\lambda_{4}$ distributions are generated mostly from a random distribution. Also possible differences in the lateral distributions of the particles measured by KASCADE and PAMIR seem too small to significantly affect the $\lambda_{4}$ distributions.

This interpretation is supported by comparing the fraction of aligned events measured by KASCADE $F\left(\lambda_{4} \geq\right.$ $0.8) \simeq(4 \pm 1) \%$ with the values derived from RUNJOB of $(3 \pm 1) \%[19]$ and from NA22 data $(\simeq 6 \%)[10,20$. Each result was found to agree to the respective background fluctuation, and despite the completely different measurement conditions, the fractions of aligned events are comparable to each other.

For a higher primary energy range, based on $\lambda_{4}$ distributions obtained from PAMIR data such as shown in 

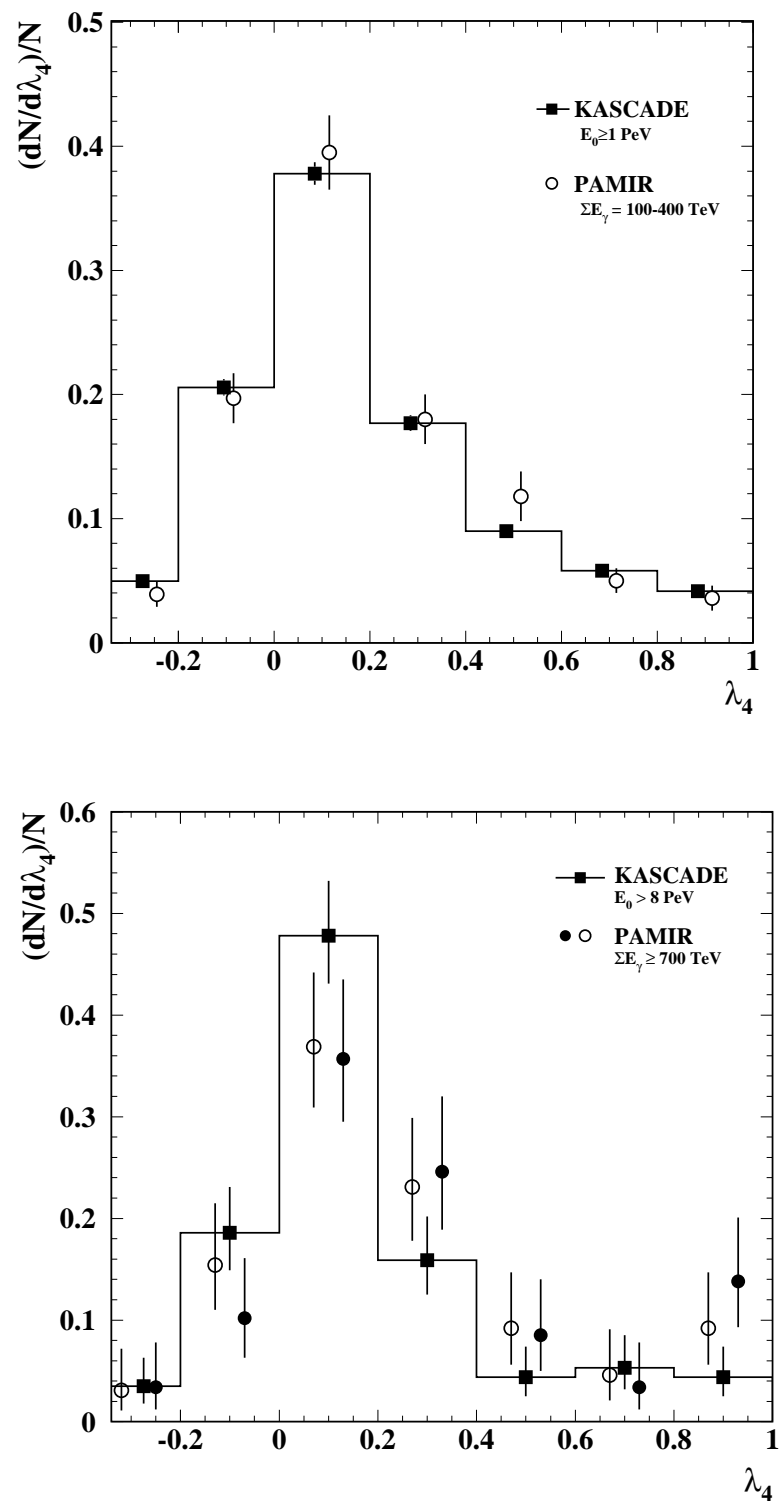

FIG. 9: $\lambda_{4}$ distributions: KASCADE and PAMIR [1, 15] data. In the lower panel, the highest primary energies are selected. The two distributions displayed for PAMIR in the lower panel refer to different reconstructions from the same PAMIR data set [11, 15]. PAMIR data are slightly displaced horizontally.
Fig. 9 (lower panel), an alignment excess with a threshold energy of $8-10 \mathrm{PeV}$ was deduced 11, 15]. Displayed in the Figure are two distributions obtained with different reconstruction methods from the same PAMIR data set. Included in the graph are the KASCADE results for primary energies above $8 \mathrm{PeV}$. Despite the completely different observation conditions, the distributions measured by both experiments agree well to each other. The fraction of events with $\lambda_{4} \geq 0.8$ is larger in the PAMIR data compared to KASCADE. However, the statistical significance for the fraction of aligned events to differ between the two experiments is small, amounting to $\simeq 1.5$ standard deviations only. Thus, also in this primary energy range the lack of significant differences between the $\lambda_{4}$ distributions, including the fraction of aligned events, suggests a random component as a dominant source of the $\lambda_{4}$ values observed by both, PAMIR and KASCADE.

The quantity $d_{4}^{\max }$, describing distances between the hadrons as measured by the KASCADE calorimeter, is sensitive to the transverse momenta in secondary hadron production and to the primary particle type. Transverse momenta in high-energy hadron interactions differing by a factor two or more from the standard values are disfavoured by the measurements.

Acknowledgments. The authors would like to thank the members of the engineering and technical staff of the KASCADE collaboration who contributed with enthusiasm and engagement to the success of the experiment. One of the authors (A.R.) kindly acknowledges helpful discussions with Prof. Maria Giller (Lodz) and the kind hospitality at the Institute of Nuclear Physics PAN (Cracow), where part of the manuscript was prepared. The KASCADE experiment is supported by the German Federal Ministry of Education and Research (05 CUAVK1/9) and was embedded in collaborative WTZ projects between Germany and Poland (POL 99/005), Romania (RUM 97/014) and Armenia (ARM 02/98). The Polish group acknowledges the support by KBN grant no. 1P03B03926 for the years 2004-2006.
[1] M. Aglietta et al., EAS-TOP Collaboration, Phys. Lett. B 460, 474 (1999).

[2] T. Antoni et al., KASCADE Collaboration, Nucl. Instr. Meth. A 513, 490 (2003).

[3] J. Engler et al., Nucl. Instr. Meth. A 427, 528 (1999).

[4] T. Antoni et al., KASCADE Collaboration, J. Phys. G: Nucl. Part. Phys. 25, 2161 (1999).

[5] J. Milke et al., KASCADE Collaboration, Acta Phys. Polon. B 35, 341 (2004).
[6] T. Antoni et al., KASCADE Collaboration, J. Phys. G: Nucl. Part. Phys. 27, 1785 (2001).

[7] F. Halzen and D.A. Morris, Phys. Rev. D 42, 1435 (1990).

[8] I. Royzen, Mod. Phys. Lett. A 9, 3517 (1994).

[9] L.T. Baradzei et al., Proceedings of Intern. Symp. on Cosmic Rays and Part. Phys., Tokyo, 136 (1984); A.S. Borisov et al., PAMIR Collaboration, Proceedings of the 22th Intern. Cosmic Ray Conf., Dublin, 4, 125 
(1991).

[10] V.V. Kopenkin, A.K. Managadze, I.V. Rabokolskaya, and T.M. Roganova, Phys. Rev. D 52, 2766 (1995).

[11] A.S. Borisov et al., Nucl. Phys. B (Proc. Suppl.) 52B, 218 (1997).

[12] S.A. Slavatinsky, Nucl. Phys. B (Proc. Suppl.) 52B, 56 (1997); S.A. Slavatinsky, Nucl. Phys. B (Proc. Suppl.) 122, 3 (2003); A.S. Borisov et al., Proceedings of the 28th Intern. Cosmic Ray Conf., Tsukuba, 85 (2003).

[13] A.S. Borisov et al., Nucl. Phys. B (Proc. Suppl.) 75A, 144 (1999).

[14] A.S. Borisov et al., Nucl. Phys. B (Proc. Suppl.) 97, 118 (2001).

[15] J.N. Capdevielle and S.A. Slavatinsky, Nucl. Phys. B (Proc. Suppl.) 75A, 12 (1999).

[16] J.N. Capdevielle, Nucl. Phys. B (Proc. Suppl.) 97, 126 (2001); J.N. Capdevielle, R. Attallah, and M.C. Talai, Proceedings of the 27th Intern. Cosmic Ray Conf., Hamburg, 1410 (2001).

[17] V.I. Osedlo et al., Proceedings of the 27th Intern. Cosmic Ray Conf., Hamburg, 1426 (2001); A.V. Apanasenko et al., Proceedings of the 15th Intern. Cosmic Ray Conf., Plovdiv, 7, 220 (1977).

[18] L. Xue et al., Proceedings of the 26th Intern. Cosmic Ray Conf., Salt Lake City, 1, 127 (1999).

[19] V.I. Galkin et al., Proceedings of the 27th Intern. Cosmic Ray Conf., Hamburg, 1407 (2001).

[20] S.M. Yandarbiev, I.V. Rakobolskaya, L.N. Smirnova, and L.G. Sveshnikova, Report No. 94-18/340, Institute of Nuclear Physics, Moscow State University, 1994 (unpublished).

[21] S.M. Troshin and N.E. Tyurin, Cosmic rays and antishadowing, hep-ph/0501018 (2005)

[22] A.R. White, Proceedings of the 8th Intern. Symp. on
Very High Energy Cosmic Ray Interactions, Tokyo, 468 (1994).

[23] R.A. Mukhamedshin, Proceedings of the 24th Intern. Cosmic Ray Conf., Rome, 1, 247 (1995); R.A. Mukhamedshin, Nucl. Phys. B (Proc. Suppl.) 97, 122 (2001).

[24] A.S. Borisov et al., PAMIR Collaboration, Izv. Akad. Nauk SSSR, ser. fiz. 49, 1285 (1985); L.T. Baradzei et al., PAMIR Collaboration, Izv. Akad. Nauk SSSR, ser. fiz. 50, 2125 (1986).

[25] V.S. Puchkov, Nuovo Cim. C 19, 1011 (1996); T.S. Yuldashbaev and K. Nuritdinov, Proceedings of the 27th Intern. Cosmic Ray Conf., Hamburg, 1415 (2001). C.R.A. Augusto et al., ibid., 1422 (2001); B.N. Kalinkin and Yu.F. Gagarin, preprint hep-ph/0109175 (2001)

[26] A. Iwan et al., KASCADE Collaboration, Proceedings of the 28th Intern. Cosmic Ray Conf., Tsukuba, Japan, 1595 (2003).

[27] D. Heck, J. Knapp, J.N. Capdevielle, G. Schatz, and T. Thouw, Report FZKA 6019, Forschungszentrum Karlsruhe (1998).

[28] N.N. Kalmykov, S.S. Ostapchenko, and A.I. Pavlov, Nucl. Phys. B (Proc. Suppl.) 52B, 17 (1997); D. Heck et al., KASCADE Collaboration, in Proceedings of the 27th Intern. Cosmic Ray Conf., Hamburg, 233 (2001).

[29] GEANT 3.21, Detector Description and Simulation Tool, CERN Program Library Long Writeups W5015, CERN (1993).

[30] A. Iwan, University of Lodz, PhD thesis (2003) (in Polish).

[31] H. Ulrich et al., Eur. Phys. J. C33, s944 (2004); T. Antoni et al., KASCADE Collaboration, Astropart. Phys., in preparation. 